\title{
Age-Dependent Alteration in Hippocampal Neurogenesis Correlates with Learning Performance of Macaque Monkeys
}

\author{
Ken AIZAWA ${ }^{1)}$, Naohide AGEYAMA ${ }^{2)}$, \\ Chihiro YOKOYAMA ${ }^{3)}$, and Tatsuhiro HISATSUNE ${ }^{1)}$ \\ ${ }^{1)}$ Department of Integrated Biosciences, The University of Tokyo, Kashiwa 277-8562, \\ ${ }^{2)}$ Tsukuba Primate Research Center, National Institute of Biomedical Innovation, Tsukuba 305-0843, \\ and ${ }^{3)}$ Functional Probe Research Laboratory, Molecular Imaging Research Program, \\ RIKEN Kobe Institute, Kobe 650-0047, Japan
}

\begin{abstract}
Newborn neurons are continuously produced in the hippocampus, which may be involved in several cognitive functions, including learning and memory, throughout life. However, both hippocampus-dependent cognitive functions and the level of adult neurogenesis are gradually attenuated as aging progresses. Few studies have explored the relationship between adult neurogenesis and cognitive functions, especially in primates. In this study, we evaluated learning performance and hippocampal neurogenesis utilizing young and aged cynomolgus monkeys. Significant attenuations in learning performance and adult neurogenesis were detected in aged monkeys. Interestingly, there was a positive correlation between learning performance and the level of neurogenesis. Our findings suggest that cognitive functions and adult neurogenesis may have some interdependent relationships during aging.
\end{abstract}

Key words: aging, hippocampal neurogenesis, primates

The hippocampus plays key roles in several cognitive functions, including learning and memory [21]. In addition, the hippocampus is one of the few regions in which newborn neurons are constantly generated from proliferating neural stem/progenitor cells throughout life $[1,6,7,24]$, and contributes to several cognitive functions $[17,19]$. However, aging causes severe attenuation in adult neurogenesis $[10,13,14,18]$. In particular, the proliferation process is severely influenced by aging, and might accompany age-dependent cognitive dysfunction $[3,4]$. Therefore, understanding the age-related decline of adult neurogenesis in cognitive impaired subjects is important for therapeutic approaches to upregulation of neurogenesis in aged brain.

In this study, we explored the relationship between the learning function and adult hippocampal neurogenesis utilizing young and aged non-human primates (macaque monkeys). The neuroanatomy and cognitive functions of macaque monkeys are very close to those of humans. Therefore, the neurobiological studies using aged macaque monkeys provide effective data for understanding age-dependent changes of neurobiological

(Received 5 December 2008 / Accepted 8 January 2009)

Address corresponding: T. Hisatsune, Department of Integrated Biosciences, The University of Tokyo, Bioscience Building 402, 5-1-5 Kashiwanoha, Kashiwa, Chiba 277-8562, Japan 
mechanisms, data which could not be obtained with other experimental animals, such as rodents.

Subjects: Three young ( 2 females and 1 male, $5.3 \pm$ 0.8 years old) and four aged ( 4 females, $22.8 \pm 0.9$ years old) cynomolgus monkeys (Macaca fascicularis) maintained at the Tsukuba Primate Research Center were studied. Several biomarkers, including the process of sexual maturation and life-span, indicate that the triplication of cynomolgus monkey age corresponds approximately to human age [23]. Therefore, the young monkeys used in this study corresponded to about 16-year-old humans, and the aged monkeys corresponded to about 70 -year-old humans. The monkeys were housed individually in cages of width $60 \mathrm{~cm}$, height $70 \mathrm{~cm}$, and depth $70 \mathrm{~cm}$ (in accordance with NIH requirements). These monkeys had not been used in any research prior to the present experiments. The monkeys were fed with apple $(100 \mathrm{~g})$ in the morning and commercial monkey chow (Type AS, Oriental Yeast, Chiba, Japan) (70 g) in the afternoon throughout the experiments. Before the behavioral test was performed, the feeding of apple was restricted, and the apple was provided for the monkeys as rewards in the testing procedures. Water was available ad libitum in the home cage. The procedures of this study were conducted in strict accordance with the Guide for the Care and Use of Laboratory Animals from the National Institutes of Health and the Guide for the Care and Use of Laboratory Animals formulated by the National Institute of Biomedical Innovation. All animal experimentation was approved by the Graduate School of Frontier Sciences, the University of Tokyo (Kashiwa, Japan) and the Animal Welfare and Animal Care Committee of the National Institute of Biomedical Innovation (Osaka, Japan).

Behavioral testing: To measure learning performance in the monkeys, we used visual pattern discrimination (VPD) tasks with minor modifications. Because this type of task has training periods which are age-dependent [15], it is appropriate for investigating the learning performance of aged monkeys. In addition, the hippocampal formation is involved in the performance of VPD tasks [11, 12]. In the current study, a pair of patterned cards was simultaneously presented to the monkey in each trial. The monkeys were required to select one of the patterned cards. In this study, the test consisted of two continuous tasks (VPD task 1 and VPD task 2). All behavioral procedures were conducted using a modified Wisconsin General Test Apparatus (WGTA) in a dark and sound-attenuated room [15].

For VPD task 1, thirteen different patterned cards (6 $\mathrm{cm}$ square) were used. We defined one card as a reward card and the other 12 cards as non-reward cards. A pair of cards was presented to a monkey in each trial. The reward card (Fig. 1A, arrows) was presented in each trial, but the combination of the cards changed randomly. During the trials, the reward card was presented equally in the left and right positions. When the monkeys selected the reward card, they were given a piece of apple as a reward. They were tested for 40 trials per day (one block), and 15 consecutive blocks were performed. Each individual task score represented the number of times that the reward cards were selected. VPD task 2 immediately followed VPD task 1 . In VPD task 2, thirteen new patterned cards were provided. All of the patterns differed from those in VPD task 1. We defined one card as a non-reward card and the other 12 cards as reward cards (Fig. 1B). The experimental procedures and quantification of score were the same as in VPD task 1.

Preparation of tissue sections: According to protocols previously described $[8,9]$, the monkeys were deeply anesthetized with ketamine hydrochloride and an overdose of sodium pentobarbital, then transcardially perfused with phosphate-buffered saline (PBS) (pH 7.4) containing heparin, followed by $4 \%$ paraformaldehyde. The brains were removed, post-fixed overnight in $4 \%$ paraformaldehyde at $4^{\circ} \mathrm{C}$, and then dissected into tissue blocks that contained the hippocampal formation. The blocks of brain tissue were placed sequentially in 10,20, and $30 \%$ sucrose in PBS at $4^{\circ} \mathrm{C}$ for one day in each solution, and then frozen at $-80^{\circ} \mathrm{C}$. The frozen brain tissue was sliced into $40-\mu \mathrm{m}$ thick coronal sections on a cryostat (Microm, Walldorf, Germany).

Immunohistochemistry: To investigate the level of neurogenesis, we performed immunohistological staining for proliferating cells, according to protocols previously described $[5,22]$. The proliferating cells in the subgranular zone (SGZ; two-cell thick region from the inner margin of the dentate granular cell layer (GCL)) were examined for an endogenous marker, Ki67, which 
(A)

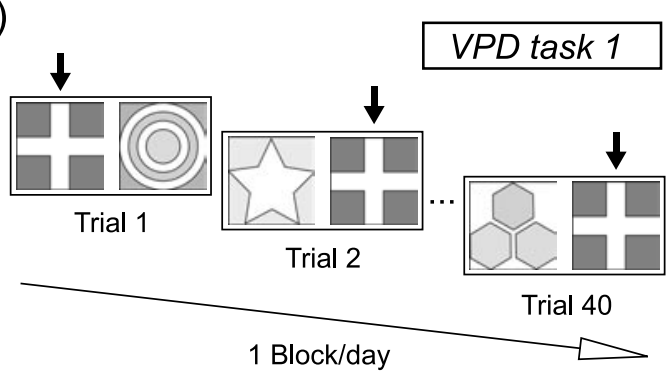

(B)

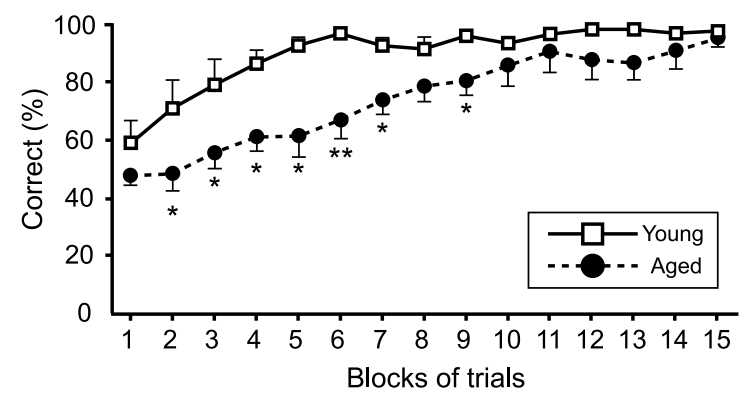

(C)

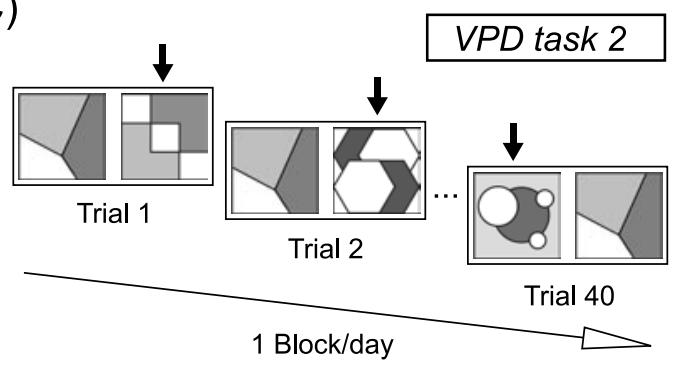

(D)

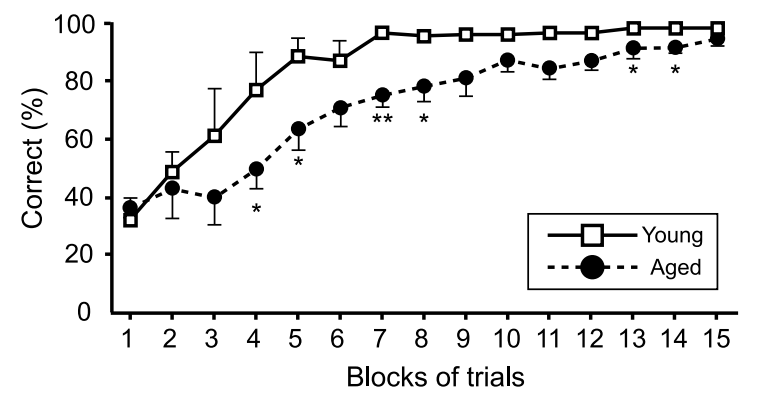

Fig. 1. Schematic diagram of the VPD tasks. (A) One reward card and 12 non-reward cards were provided in VPD task 1. (B) One non-reward card and 12 reward cards were provided in VPD task 2. Arrows indicate the reward cards. (C) Learning curve for the VPD task 1 in the young monkeys and the aged monkeys. (D) Learning curve for the VPD task 2 in the young monkeys and the aged monkeys. ${ }^{*} P<0.05,{ }^{* *} P<0.01$.

is expressed in the cells within the dividing cycle [22]. Free-floating sections were incubated with mouse monoclonal Ki67 antibody (1:50, Novocastra, Newcastle, UK) diluted in Tris-buffered saline (TBS)/0.5\% Triton X-100 for 3 days at $4{ }^{\circ} \mathrm{C}$. After the primary staining, the sections were washed in TBS, and then incubated with secondary immunofluorescent antibodies, Alexa 488 (1:1,000, Molecular Probes, Eugene, OR), for $2 \mathrm{~h}$ at room temperature. The stained sections were also incubated with 4', 6-diamidino-2-phenylindole (DAPI, 1:1,000, Sigma, St. Louis, MO) to visualize the area of the dentate gyrus.

Stereological analysis: The immunohistologically marked cells were counted in the SGZ with stereology. With a confocal laser scanning microscope (TCS SP2; Leica, Mannheim, Germany), Ki67 ${ }^{+}$cells were counted in every 13th section (520 $\mu \mathrm{m}$ apart) through the entire antero-posterior extent of the hippocampus. All the immunolabeled cells were counted in each section by an experimenter blinded to the study code. The putative number of marked cells in the SGZ was tallied and multiplied by the section sampling fraction.
Statistical analysis: Data were expressed as the mean \pm SEM. The statistical significance of the difference between means was assessed by Student's $t$-test, with the level of significance being set at $P<0.05$ and $P<0.01$. Relationships between the behavioral score and the number of labeled cells were evaluated using Pearson's correlation test.

Behavioral characterization: In the VPD task 1, both the young and the aged monkeys showed the ability to learn the task, but the latter showed significant delays in learning the task (Fig. 1C). The VPD task 2 immediately followed the VPD task 1 . The monkeys were required to select the one reward card in the VPD task 1, while they were required not to select the one non-reward card in the VPD task 2. This was a type of set-shifting task. This changing protocol allowed the addition of quasi-new trials without additional training. As in the VPD task 1, the young monkeys learned the task in the early blocks of the task, but the aged monkeys required a larger number of blocks to learn the task (Fig. 1D). The aged monkeys eventually learned the task, but 

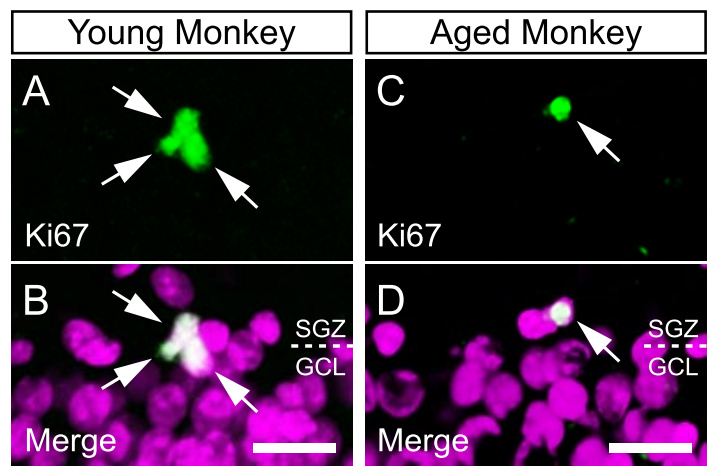

(E)

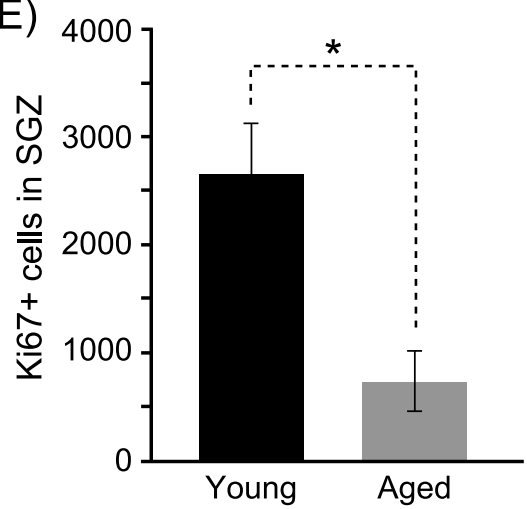

Fig. 2. Proliferating cells in the SGZ of monkeys. (A, B) Proliferating cells labeled for Ki67 (A, green) were frequently found in the young monkeys. (C, D) In contrast, in the aged monkeys, few proliferating cells were observed in the SGZ (B, green). Arrows indicate proliferating cells labeled for Ki67. DAPI staining is shown in magenta. GCL, granular cell layer; SGZ, subgranular zone. (E) There was a statistical difference in the number of proliferating cells between the young and aged monkeys. ${ }^{*} P<0.05$. Scale bars, $20 \mu \mathrm{m}$.

showed significantly slower learning than the young monkeys in both VPD tasks. These results indicate that learning performance significantly deteriorates in aged monkeys despite the preservation of the memory function itself. Moreover, the results suggest that aged monkeys are an appropriate animal model of age-related cognitive dysfunction.

Hippocampal neurogenesis: Proliferating cells in the young SGZ were frequently located in group clusters (Figs. 2A and 2B). By contrast, few proliferating cells labeled for Ki67 were observed in the aged monkeys (Figs. 2C and 2D). The stereological analysis revealed that there was a significant reduction in the number of proliferating cells in the SGZ between the young and

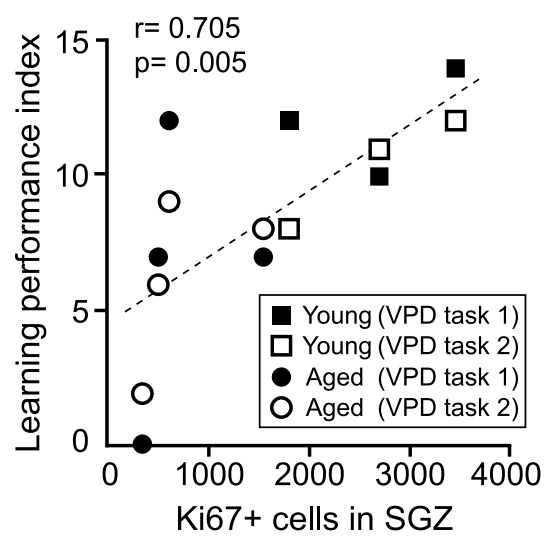

Fig. 3. Correlation between individual learning performance of the VPD tasks and the number of proliferating cells in the SGZ. There was a positive relationship (dotted line) between the learning performance index and the number of $\mathrm{Ki}^{+} 7^{+}$cells. Closed and open squares indicate the learning performance index of the young monkeys in the VPD task 1 and the VPD task 2, respectively. Closed and open circles indicate the learning performance index of the aged monkeys in the VPD task 1 and the VPD task 2, respectively.

aged monkeys (Fig. 2E, $P<0.01$ ). This result demonstrates that a significant age-related attenuation in hippocampal neurogenesis occurs in the primate SGZ.

Correlation between learning performance and hippocampal neurogenesis: To explore the possibility that there is a relationship between the cognitive function and the level of adult neurogenesis during aging, we then analyzed the correlation between the score of VPD tasks and the number of proliferating cells in the SGZ. The scores of the VPD tasks were assessed as a learning performance index. The learning performance index was calculated as: 15-(block number when the monkey first achieved an $80 \%$ correct task score in the VPD task). Therefore, the learning performance index could have a value from 0 (impaired) through 15 (unimpaired). We found a positive correlation between the learning performance index and the number of proliferating cells in the SGZ (Fig. 3). Interestingly, in the young group, the monkeys with good learning performance exhibited a higher level of hippocampal neurogenesis compared to the monkeys showing more impaired performances. However, in the aged group, this tendency was not ob- 
served and there was considerable individual variability of learning performance. The observation of individual differences in the learning performance of aged monkeys was in agreement with a previous study [15]. Taken together, our present data suggest that adult neurogenesis could be involved in learning functions, but this relationship might be attenuated during aging .

The hippocampal formation, especially the dentate gyrus, is particularly vulnerable to aging which might explain cognitive decline during aging $[2,16,20]$. Because a VPD task requires the hippocampal formation $[11,12]$, the age-related neurobiological alterations in the hippocampus, including changes in the neurogenesis process, may induce learning dysfunctions in aged primates. Understandably, other brain regions, such as the visual and prefrontal cortices, also relate to performance of VPD tasks; the visual cortex is related to the ability to discriminate of the patterns drawn on cards, and the prefrontal cortex is involved in the flexibility and attention needed in learning behavior [2]. Thus, the attenuation of hippocampal neurogenesis might be influenced by general attenuation of neuronal plasticity, not by hippocampus-specific alteration.

In conclusion, aging caused severe deterioration of learning performance, which was easily detected with the modified VPD tasks. In addition, we demonstrated that the level of adult neurogenesis in the hippocampus was significantly attenuated during aging. Interestingly, there was a positive correlation between the age-dependent attenuations in learning performance and adult neurogenesis. It is possible that there are interdependent relationships between adult neurogenesis and cognitive functions, including learning performance. The agerelated deterioration of learning performance might be related to the levels of hippocampal neurogenesis.

\section{Acknowledgment(s)}

This work was supported by a Grant-in-Aid for Scientific Research from the Ministry of Education, Culture, Sports, Science and Technology of Japan. We thank Drs. Djoher Nora Abrous and Elodie Drapeau for informative discussions and insightful comments. We acknowledge the excellent animal care support of Drs. Fumiko Ono, Naoyuki Saito, Yuko Katakai, Hiromi Ogawa, and Haya- to Narita at the Tsukuba Primate Research Center.

\section{References}

1. Abrous, D.N., Koehl, M., and Le Moal, M. 2005. Physiol. Rev. 85: 523-569.

2. Burke, S.N. and Barnes, C.A. 2006. Nat.Rev. Neurosci. 7 : $30-40$.

3. Drapeau, E., Mayo, W., Aurousseau, C., Le Moal, M., Piazza, P.V., and Abrous, D.N. 2003. Proc. Natl. Acad. Sci. U.S.A. 100: 14385-14390.

4. Drapeau, E., Montaron, M.F., Aguerre, S., and Abrous, D.N. 2007. J. Neurosci. 27: 6037-6044.

5. Fukuda, S., Kato, F., Tozuka, Y., Yamaguchi, M., Miyamoto, Y., and Hisatsune, T. 2003. J. Neurosci. 23: 9357-9366.

6. Gage, F.H. 2000. Science 287: 1433-1438.

7. Kempermann, G., Jessberger, S., Steiner, B., and Kronenberg, G. 2004. Trends Neurosci. 27: 447-452.

8. Koketsu, D., Furuichi, Y., Maeda, M., Matsuoka, N., Miyamoto, Y., and Hisatsune, T. 2006. Exp. Neurol. 199: 92-102.

9. Koketsu, D., Mikami, A., Miyamoto, Y., and Hisatsune, T. 2003. J. Neurosci. 23: 937-942.

10. Kuhn, H.G., Dickinson-Anson, H., and Gage, F.H. 1996. J. Neurosci. 16: 2027-2033.

11. Mahut, H., Zola-Morgan, S., and Moss, M. 1982. J. Neurosci. 2: 1214-1229.

12. Moss, M., Mahut, H., and Zola-Morgan, S. 1981. J. Neurosci. 1: $227-240$.

13. Olariu, A., Cleaver, K.M., and Cameron, H.A. 2007. J. Comp. Neurol. 501: 659-667.

14. Rao, M.S., Hattiangady, B., and Shetty, A.K. 2006. Aging Cell 5: 545-558.

15. Rapp, P.R. 1990. Behav. Neurosci. 104: 876-884.

16. Rosenzweig, E.S. and Barnes, C.A. 2003. Prog. Neurobiol. 69: 143-179.

17. Saxe, M.D., Battaglia, F., Wang, J.W., Malleret, G., David, D.J., Monckton, J.E., Garcia, A.D.R., Sofroniew, M.V., Kandel, E.R., Santarelli, L., Hen, R., and Drew, M.R. 2006. Proc. Natl.Acad.Sci. U.S.A. 103: 17501-17506.

18. Seki, T. and Arai, Y. 1995. Neuroreport 6: 2479-2482.

19. Shors, T.J., Miesegaes, G., Beylin, A., Zhao, M., Rydel, T., and Gould, E. 2001. Nature 410: 372-376.

20. Small, S.A., Chawla, M.K., Buonocore, M., Rapp, P.R., and Barnes, C.A. 2004. Proc. Natl. Acad. Sci. U.S.A. 101: 7181-7186.

21. Squire, L.R. 2004. Neurobiol. Learn. Mem. 82: 171-177.

22. Tozuka, Y., Fukuda, S., Namba, T., Seki, T., and Hisatsune, T. 2005. Neuron 47: 803-815.

23. Yoshida, T., Ofuji, H., and Ono, F. 2006. pp. 47-61. In: The TPRC Handbook on the Care and Management of the Laboratory Cynomolgus Monkey (Yoshida, T. and Fujimoto, K. eds.), Springer Japan, Tokyo (in Japanese).

24. Zhao, C., Deng, W., and Gage, F.H. 2008. Cell 132: 645660. 